\title{
Extração de vias combinando métodos de detecção de regiões e linhas em imagens de intensidade de pulso laser
}

\author{
Tatiana Sussel Gonçalves MENDES ${ }^{1}$ \& Aluir Porfírio DAL POZ \\ ${ }^{1}$ Programa de Pós-Graduação em Ciências Cartográficas, Universidade Estadual Paulista, Rua Roberto Simonsen, 305, CEP \\ 19060-900. Presidente Prudente, SP, Brasil.E-mail: tatisussel@gmail.com \\ ${ }^{2}$ Departamento de Cartografia, Universidade Estadual Paulista, Rua Roberto Simonsen, 305, CEP 19060-900, Presidente \\ Prudente, SP, Brasil.E-mail: aluir@fct.unesp.br
}

Recebido em 07/2010. Aceito para publicação em 08/2011.

Versão online publicada em 05/11/2012 (www.pesquisasemgeociencias.ufrgs.br)

\begin{abstract}
Resumo- Este trabalho tem por objetivo extrair os eixos de vias a partir de regiões de vias isoladas previamente, tendo por base uma imagem de intensidade de pulso laser. Neste tipo de imagem, as vias são facilmente identificadas, pois se apresentam como faixas alongadas e em tonalidade bem escura e homogênea, contrastando com os outros objetos da imagem. A imagem de intensidade é segmentada através da técnica de crescimento de regiões, gerando regiões que representam as vias. A partir dessas regiões, os eixos das vias são extraídos de duas formas. Uma delas combina o método de detecção de linhas de Steger com um procedimento de limiarização por comprimento das linhas, visando a reduzir a ocorrência de linhas espúrias. A outra se baseia no método de esqueletização de regiões pela Transformada do Eixo Médio, seguido de um processo de poda para eliminar o máximo possível as ramificações. A partir dos experimentos realizados é possível comparar os eixos extraídos pelas duas formas descritas, e aquela que utiliza o método de detecção de linhas de Steger apresenta melhores resultados do que a baseada no método de esqueletização.
\end{abstract}

Palavras-chave: extração de vias, segmentação, detecção de linhas, esqueletização.

\begin{abstract}
STREET EXTRACTION COMBINING METHODS FOR REGION AND LINE DETECTION FROM LASER SCANNING INTENSITY IMAGES. This paper aims at extracting street centerlines from previously isolated street regions by using the image of laser scanning intensity. In this image, streets are easily identified, since they manifest as dark, elongate ribbons contrasting with background objects. The intensity image is segmented by using the region growing technique, which generates regions representing the streets. From these regions, the street centerlines are extracted in two manners. The first one is through the Steger lines detection method combined with a line length thresholding by which lines being shorter than a minimum length are removed. The other manner is by combining the skeletonization method of regions based on the Medial Axis Transform and with a pruning process to eliminate as much as possible the ramifications. Experiments showed that the Steger-based method provided results better than the method based on skeletonization.
\end{abstract}

Keywords: street extraction, segmentation, detection of lines, skeletonization.

\section{Introdução}

Pesquisas cujo foco é a extração automática de rodovias têm sido estimuladas para simplificar o processo de aquisição e de atualização de base de dados em Sistemas de Informações Geográficas (SIG). Comparado com um número relativamente alto de grupos de pesquisas que focam seus trabalhos em extração de rodovias em áreas rurais, poucos grupos atuam na extração automática de rodovias em ambientes urbanos (Samadzadegan et al., 2009). Segundo Dal Poz (2001), isto ocorre porque, em cenas rurais, o objeto rodovia pode ser estudado individualmente, ao contrário das cenas urbanas, onde é praticamente impossível modelar a malha viária sem considerar o contexto, ou seja, a relação existente entre rodovias (vias) e outros objetos (edificações, árvores, veículos, sombras etc.). Por sua vez, as informações contextuais são de difícil modelagem.

Para lidar com a alta complexidade dos ambientes urbanos, alguns trabalhos usam modelos de vias mais complexos e informações adicionais. Hinz \& Baumgartner (2003), usando imagens de alta-resolução, integram informações sobre as características radiométricas, geométricas e topológicas, e usam como informação adicional um MDS (Modelo Digital de Superfície). A integração de imagens coloridas com a informação pré-existente de uma base digital de dados espaciais pode ser encontrada em Zhang (2004). Com o surgimento dos sistemas de varredura a laser, 
a combinação dos dados provenientes deste sistema com imagens aéreas ou orbitais de alta resolução é uma possibilidade para facilitar a tarefa de extração da malha viária. Trabalhos relacionados podem ser encontrados em $\mathrm{Hu}$ et al. (2004) e Zhu et al. (2004), que integram imagens aéreas e dados laser, e em Tiwari et al. (2009), que integram imagens IKONOS e dados de altimetria provenientes de varredura a laser.

Alguns métodos usam somente dados de varredura a laser no processo de extração da malha viária. Nestes casos, além da informação de altura, também a informação de intensidade do pulso laser pode ser utilizada. Alharthy \& Bethel (2003) usam a intensidade do pulso laser para detectar pixels candidatos às vias e dados de altura para remover ruídos não relacionados às vias. Clode et al. (2004) usam uma técnica de classificação hierárquica para classificar os pontos laser em pontos de rodovia e não rodovia. As vias são classificadas baseando-se num intervalo de altura onde supostamente devem ser encontradas e num intervalo de intensidade do pulso laser de acordo com o tipo de material das vias. A detecção e a vetorização da malha viária é então completada em Clode et al. (2007), cujo resultado da classificação apresentada é uma imagem binária que é vetorizada através da convolução com um disco PCD (Phase Coded Disk), que fornece três informações sobre a malha viária: o eixo central, a direção e a largura.

As vias em imagens de intensidade de pulso laser aparecem em tonalidade bem escura, fato decorrente da baixa reflexão (aproximadamente 17\%) do pulso laser (Wehr \& Lohr, 1999). Por um lado, as vias podem ser facilmente identificadas, mas, por outro, vários elementos, como a vegetação, apresentam respostas parecidas. Consequentemente, a aplicação de técnicas de processamento de imagens nesses dados geralmente produz resultados bastante ruidosos. Provavelmente por isso é muito difícil encontrar métodos para a extração de vias baseados apenas em imagem de intensidade de pulso laser. Sua utilização é normalmente combinada com dados de altura laser, visando à redução de ruídos associados com elementos situados acima do terreno, tais como árvores e telhados. Este artigo mostra que é possível utilizar técnicas de processamento de imagem digitais para extrair vias em imagens de intensidade de retorno de pulso laser com um reduzido nível de ruído.

Este trabalho está organizado em 5 seções. $\mathrm{Na}$ seção 2 são apresentados os fundamentos teóricos para o desenvolvimento dos métodos. A seção 3 descreve os métodos. Os experimentos e resultados são apresentados e discutidos na seção 4. A seção 5 traz as principais conclusões.

\section{Fundamentos teóricos}

\subsection{Suavização Anisotrópica}

Os filtros gaussianos são isotrópicos, ou seja, suavizam a imagem em todas as direções, causando um borramento nas bordas. Para contornar esse problema, e tendo em vista a característica anisotrópica das bordas, Malik \& Perona (1990) desenvolveram um modelo de suavização seletiva da imagem que realiza a difusão não linear da imagem e consiste em um algoritmo para a detecção de bordas e realce de imagem. Com base neste modelo, Barcelos et al. (2003) desenvolveram uma equação de difusão não linear (anisotrópica) que permite suavizar adequadamente a imagem, preservando as bordas e outros detalhes importantes, e é dada pela Equação Diferencial Parcial (Equação 1) (Vale et al., 2004).

$u_{t}=\bar{g}|\nabla u| \operatorname{div}\left(\frac{\nabla u}{|\nabla u|}\right)-\lambda(1-\bar{g})(u-I)$

$u(x, y, 0)=I(x, y), x \in \mathfrak{R}^{2}$

onde:

div

é o operador divergente;

$\nabla$

representa o operador gradiente;

$|\nabla u| \operatorname{div}\left(\frac{\nabla u}{|\nabla u|}\right)$

é o termo que realiza o processo de difusão na imagem;

\section{$0 \leq \lambda \leq 1$}

é um escalar que controla a taxa de difusão e atua como peso para o termo $(1-\bar{g})$;

I

representa a imagem a ser processada;

$u$

é a imagem suavizada a partir de $I$ no instante $t$;

$\bar{g}=\frac{1}{1+k \mid \nabla\left(\left.G_{t}{ }^{*} u\right|^{2}\right.}, \mathrm{com} \quad 0 \leq \bar{g} \leq 1$

é um termo que controla a intensidade da difusão; 
$k$

é uma constante presente na função $\bar{g}$, e $G_{t}$ é uma função gaussiana.

O modelo de suavização seletiva da imagem (Equação 1) é composto de dois termos: o primeiro, $\bar{g}|\nabla u| \operatorname{div}\left(\frac{\nabla u}{|\nabla u|}\right)$

é um termo de difusão que permite a eliminação dos ruídos da imagem inicial $I$. 0 segundo, $\lambda(1-\bar{g})(u-I)$, é um termo "forçante" que força a imagem $u$ a permanecer próxima da imagem inicial I. A função dada acima é uma das funções que foram propostas (Malik \& Perona, 1990) para controlar a intensidade da difusão. Sua ação em regiões de baixo e de alto contraste se dá na forma a seguir (Vale et al., 2004):

Quando $\left|\nabla\left(G_{t} * u\right)\right|$ for pequeno, tem-seque $k\left|\nabla\left(G_{t}^{*} u\right)\right|$ presente na função $\bar{g}$ será muito pequeno. Nestas condições $\bar{g} \sim 1$, fazendo com que o termo $(1-\bar{g}) \sim 0$ na Equação 1 . Desta forma, o termo $(u-I)$ que mantém as bordas inalteradas não atuará no modelo e como consequência, o processo de difusão efe-tuado no primeiro termo na Equação 1 fará com que a região homogênea sofra maior suavização.

Quando $\left|\nabla\left(G_{t}^{*} u\right)\right|$ for grande, ou seja, se houver variações bruscas nos níveis de cinza da imagem, $k\left|\nabla\left(G_{t}{ }^{*} u\right)\right|$ presente no denominador da função $\bar{g}$ será muito grande, logo $\bar{g} \sim 0$, o que implica em $(1-\bar{g}) \sim 1$ na Equação 1. Neste caso, o termo $(u-I)$ atuará significativamente na imagem, mantendo as características originais das bordas. Isso faz com que o processo de difusão efetuado pelo primeiro termo na Equação 1 não atue nessas regiões.

A função gaussiana $G_{t}$ presente na Equação 1 foi ligeiramente modificada em relação à função gaussiana original, fazendo $\sigma^{2}=a t$. Nesta expressão, $\sigma$ é o desvio-padrão da gaussiana e $a$ é uma constante positiva. A função gaussiana $G_{t}$ é dada por:

$$
G_{t}(x, y)=\frac{1}{2 a \pi t} e^{\frac{-\left(x^{2}+y^{2}\right)}{2 a t}},(x, y) \in \mathfrak{R}^{2}
$$

Logo, se fosse possível estimar o nível de ruído ou de informação irrelevante, o que seria representado por $\sigma^{2}$, seria também possível evoluir, num processo iterativo, até um grau de suavização ótimo (T), dado por (Barcelos et al., 2003):

$$
T=\frac{\sigma^{2}}{a}
$$

Sendo $\Delta t$ o tamanho do passo de evolução temporal e levando em conta que o valor de $T$ é normalmente desconhecido, o parâmetro $t$ iniciaria com um valor inicial $t_{0}$, passando sucessivamente por $t_{0}+\Delta t, t_{0}+2 \Delta t, \ldots$, até atingir o nível desejado de suavização. 0 valor de $t$ correspondente ao nível desejado de suavização corresponderia ao tempo ótimo de suavização $(T)$.

\subsection{Detector de Linhas de Steger}

Importantes feições lineares, como rios, rodovia e vias são exemplos de linhas em imagens digitais. Estas feições são estruturas curvilíneas do tipo $s(t)$ que podem ser caracterizadas pelo atributo intensidade (como por exemplo, tons de cinza) em um perfil perpendicular a ela (Steger, 2000). No caso, como as rodovias ou vias em imagens digitais são linhas ou faixas estreitas de alta intensidade de brilho, perfis transversais das mesmas podem ser adequadamente representados pelo modelo de linha mostrado na figura 1.



Figura 1. (A) Perfil de linha e (B) equação que determina o perfil de linha (Adaptado de Steger, 2000).

Numa imagem digital, uma linha pode ser modelada como uma curva $s(t)$, que apresenta na direção transversal um comportamento característico de perfil unidimensional dado por $f_{a}(x)$ mostrado na figura 1. Portanto, deve-se analisar a direção transversal à linha em um dado ponto $P$ da curva $s(t)$, correspondente ao vetor normal $\vec{n}(t)$ e assim, extrair pontos ao longo das linhas utilizando a primeira derivada direcional na direção $\vec{n}(t)$. Logo, o problema se resume a determinar a direção $\vec{n}(t)$ em cada ponto da linha. Essa direção pode ser obtida a partir do autovetor $\left(n_{x^{\prime}} n_{y}\right)$ correspondente ao autovalor de maior magnitude da matriz hessiana (Equação 4) da imagem suavizada (Steger, 1998).

$H(x, y)=\left(\begin{array}{ll}r_{x x} & r_{x y} \\ r_{x y} & r_{y y}\end{array}\right)$

onde, $r_{x x}$ é a derivada segunda em relação à direção $x, r_{y y}$ é a derivada segunda em relação à direção $y$ 
e $r_{x y}$ é a derivada mista, todos tomados sobre a imagem suavizada.

Os autovalores $(\lambda)$ de $H(x, y)$ podem ser determinados através de $\operatorname{det}(H(x, y)-I \lambda)=0$, onde det é o determinante matricial. A partir dos autovalores, é possível determinar os autovetores que fornecem a direção transversal à linha $\left(n_{x}, n_{y}\right)$, sendo eles obtidos através da solução $(X)$ de $(H(x, y)-I \lambda)=0$ (Ishibashi, 2008).

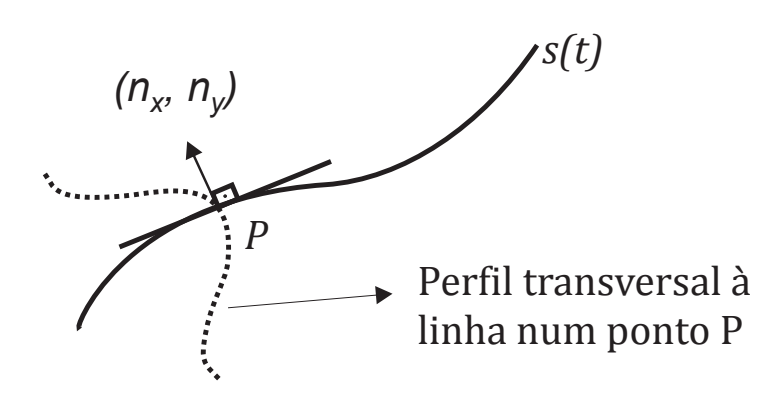

Figura 2. Perfil transversal à linha num ponto $P$ modelado através da expansão de Taylor.

A partir do autovetor $\left(n_{x} n_{y}\right)$ é possível modelar, com uma expansão de Taylor de segunda ordem, o perfil transversal a uma linha em um dado ponto $P$ (Fig. 2). Para determinar a melhor localização do ponto de linha no perfil transversal em análise, deve-se determinar onde a primeira derivada na direção $\left(n_{x} n_{y}\right)$ se anula. Este ponto pode ser obtido inserindo $\left(t n_{x}, n_{y}\right)$ no polinômio de segunda ordem resultante da expansão de Taylor e, após, obter um valor $t^{\prime}$ de $t$ que minimize o polinômio (Steger, 1998). As coordenadas ( $t^{\prime} n_{x^{\prime}} t^{\prime} n_{y}$ ) correspondem a um ponto da linha com precisẫo subpixel. Para validar este ponto, a derivada segunda do polinômio em $t^{\prime}$ deve, segundo um limiar, ser muito grande para linhas claras, ou muito pequeno para linhas escuras. Este limiar pode ser determinado automaticamente através da análise de um histograma da segunda derivada, visto que os pontos de linha bem definida na imagem corresponderão a valores altos em módulo da segunda derivada e, como poucos pontos da imagem pertencerão às linhas, terão baixa frequência. Todo esse processo deve ser repetido para todos os pontos (Ishibashi, 2008).

\subsection{Esqueletização}

Blum (1967) foi quem primeiramente introduziu o conceito de esqueletização, definindo o esqueleto de uma região em termos da Transformada do Eixo Médio (TEM). Numa região $R$ com borda $B$, para cada ponto $p$ pertencente a esta região $R$, encontra-se o seu vizinho mais próximo na borda $B$. Se $p$ tiver mais de um vizinho a uma distancia mínima, tem-se que $p$ pertence ao eixo médio $S$ (esqueleto) da região $R$. Para encontrar o vizinho mais próximo, utiliza-se a medida de distância, e logo, o resultado de uma TEM é influenciado pela medida de distância adotada. A medida de distância mais usada é a euclidiana, que produz a menor distância real entre dois pontos (Gonzalez \& Woods, 2008).

0 esqueleto de uma região específica, usando a distância euclidiana, corresponde a um conjunto dos centros de círculos máximos inscritos nela e bitangentes à sua forma, ou seja, a curva deve tocar sua borda em pelo menos dois pontos distintos (Gonzalez \& Woods, 2008). A figura 3 mostra o esqueleto de uma região sendo gerado a partir de alguns círculos máximos e seus centros, obtidos através da TEM.

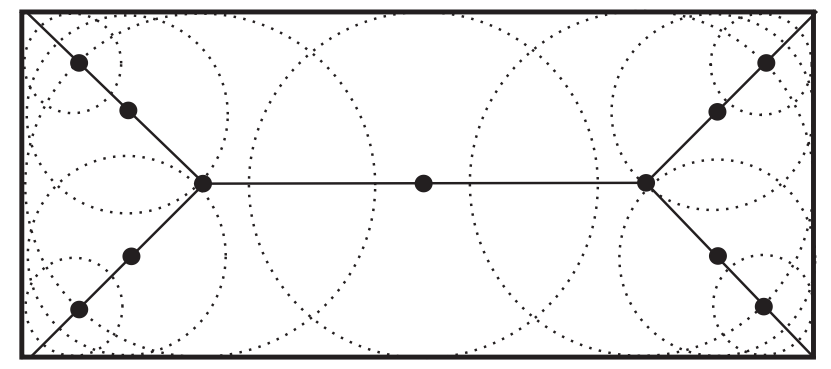

Figura 3. Esqueleto de uma região retangular definido por círculos máximos bitangentes à borda da região (Adaptado de Plotze \& Bruno, 2004).

Para complementar o processo de esqueletização, o método de poda (pruning) é de fundamental importância, principalmente em algoritmos baseados em TEM, que são sensíveis às variações locais, sendo que até mesmo o ruído de um pixel na borda de um objeto pode alterar o formato do esqueleto da região. 0 objetivo deste processo é extrair a partir de uma região esqueletizada os pontos extremos, ou seja, as ramificações. Segundo Soille (1999), uma poda de tamanho $n$ consiste em remover $n$ pixels de cada ramificação do esqueleto, a partir de cada ponto final.

\section{Materiais e métodos}

As técnicas de análise de imagens apresentadas na seção 2 foram combinadas e aplicadas na imagem intensidade do pulso laser de modo a extrair regiões que correspondem às regiões de vias e, em seguida, a detectar o eixo médio dessas vias.

Aplicou-se, inicialmente, um filtro de suavi- 
-zação de difusão anisotrópica. Em seguida, a imagem suavizada foi segmentada por crescimento de regiões e um processo de seleção de regiões foi aplicado, excluindo-se as regiões que possuem comprimento de contorno menor que um limiar pré-estabelecido. A seguir, foram aplicados na imagem segmentada dois métodos para a detecção do eixo médio das vias. 0 primeiro foi o método de detecção de linhas de Steger seguido de uma limiarização por comprimento para eliminar as linhas espúrias. 0 segundo foi o método de detecção do esqueleto de uma região (esqueletização) seguido de um procedimento de poda, que permite eliminar as ramificações características deste método. A figura 4 apresenta o fluxograma dos métodos propostos.



Figura 4. Fluxograma mostrando as etapas e as técnicas usadas para detectar vias como regiões e extrair seu eixo médio.

Todas essas etapas foram realizadas usando o software HALCON 7.1 desenvolvido pela MVTec, que é uma ampla biblioteca de ferramentas, com um ambiente de desenvolvimento integrado (IDE - Integrated Development Environment) para visão computacional.

\subsection{Pré-Processamento da Imagem}

Um pré-processamento da imagem foi realizado aplicando um filtro de modelo de difusão anisotrópica, com o objetivo de suavizar as regiões homogêneas preservando as bordas dos objetos presentes na imagem. Vale ressaltar que a locali- zação da borda não é um aspecto crítico no contexto do método proposto. Isto decorre da própria natureza da imagem de intensidade de pulso laser, onde as bordas geralmente não apresentam, de forma muito acurada, os limites reais das vias. Entretanto, a possibilidade de realizar uma acentuada regularização das regiões mais homogêneas, tornando-as bastante achatadas, sem a pulverização das bordas que delimitam as regiões, deverá possibilitar ao método de segmentação (subseção 3.2) a obtenção de segmentos menos fragmentados, principalmente os referentes às vias. A qualidade da segmentação impacta diretamente a etapa seguinte de extração de linhas, seja pelo método de Steger ou 
por esqueletização.

0 método de difusão anisotrópica depende de vários parâmetros, tais como o desvio-padrão (sigma), o tamanho do passo de evolução temporal $\Delta t$ e o número de iterações. 0 valor para a constante $k$ é fixado como sendo igual 1 . 0 parâmetro sigma determina a intensidade da suavização. Como o processo de suavização é iterativo e controlado pela evolução temporal, é necessário fornecer o número de iterações e o parâmetro $\Delta t$. À medida que o processo iterativo evolui, a imagem vai sendo progressivamente suavi- zada.

A figura 5 mostra o resultado da suavização anisotrópica de um recorte de uma imagem de intensidade de pulso laser usando diferentes números de iterações. Os parâmetros sigma e $\Delta t$ foram iguais em ambos os exemplos, sendo respectivamente 1,0 e 0,5, enquanto os números de iterações foram 50 e 100, para as figuras 5B e 5C, respectiva-mente. É possível verificar que aumentando o número de iterações, mais suavizadas se tornam as regiões e, no entanto, as bordas das vias permanecem preservadas.



Figura 5. Resultado da aplicação do filtro de suavização anisotrópica: (A) imagem de intensidade de pulso laser original; (B) imagem suavizada com 50 iterações e (C) imagem suavizada com 100 iterações.

Na verdade, a escolha de parâmetros corretos é difícil e depende do tipo de aplicação subsequente, o que envolve experimentos baseados em tentativas e erros.

\subsection{Segmentação}

A técnica de segmentação usada foi a de crescimento de regiões, que segundo Gonzalez \& Woods (2008), é uma técnica que agrupa pixels ou sub-regiões em regiões maiores com base num critério de similaridade pré-definido. É um processo iterativo e é finalizado quando nenhum pixel adjacente satisfaz o critério de inclusão em cada região.

A imagem suavizada na etapa anterior (seção 3.1) é segmentada em regiões de mesma intensidade dividindo-a em retângulos de dimensão linha $x$ coluna, que são parâmetros que devem ser fornecidos pelo usuário. Os parâmetros linha e coluna possuem a restrição de serem números ímpares, uma vez que apenas o nível de cinza do ponto central de cada retângulo é considerado para decidir se dois retângulos adjacentes pertencem à mesma região.

Assim, considerando $g_{1}$ e $g_{2}$ como níveis de cinza do ponto central de dois retângulos adjacentes, esses retângulos serão unidos em uma mesma região se a diferença dos níveis de cinza for menor ou igual ao parâmetro tolerância, conforme a expressão 5.

$\left|g_{1}-g_{2}\right|<$ tolerância

O parâmetro tolerância possui bastante influência no resultado final da segmentação. Valores pequenos trazem melhores resultados, pois unem retângulos com valores de níveis de cinza bem próximos, enquanto que valores altos podem unir retângulos com uma grande diferença entre os níveis de cinza. As regiões resultantes são coleções de retângulos de dimensão linha x coluna escolhida, e somente as regiões que contêm um tamanho mínimo de pontos são retornadas. Logo, a escolha do parâmetro tamanho mínimo influencia no tamanho e na quantidade das regiões resultantes, como pode ser observado na figura 6 . Valores pequenos $(10$, por exemplo) causam uma segmentação excessiva na imagem, ou seja, resulta em diversas regiões menores com o mínimo de 10 retângulos (Fig. 6B), enquanto ao adotar um valor maior (100, por exemplo) para se formar uma região serão necessários no mínimo 100 retângulos, obtendo-se assim uma menor quantidade de regiões (Fig. 6C).

\subsection{Seleção de Regiões de Vias}

$\mathrm{Na}$ imagem segmentada, as regiões que 


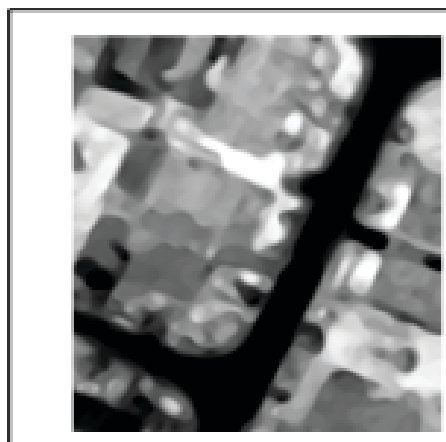

(A)

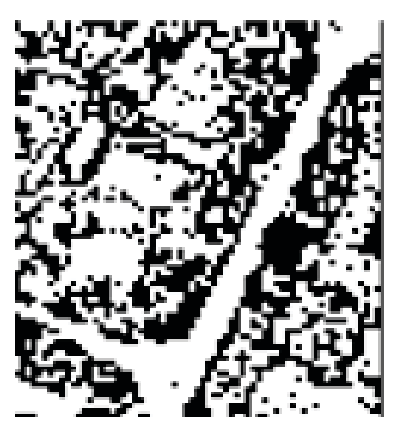

(B)



(C)

Figura 6. Influência da escolha do valor para o parâmetro tamanho mínimo: (A) imagem a ser segmentada; (B) segmentação usando tamanho mínimo = 10 e (C) segmentação usando tamanho mínimo = 100 .

representam as vias, de uma forma geral, apresentam-se alongadas e contínuas. Com o intuito de selecionar as regiões de vias aplica-se uma limiarização usando o comprimento de contorno das regiões. Essa limiarização elimina as menores cadeias de pixels que contornam as regiões segundo um limiar estabelecido.

Nesta etapa, dois parâmetros, mínimo e máximo, devem ser escolhidos, que são limiares para a dimensão dos contornos de regiões que devem permanecer. 0 intuito aqui é de eliminar regiões menores, que não são de interesse, ou seja, que não correspondem às vias. A dificuldade encontrada é que nem sempre as regiões indesejadas são as que possuem comprimentos de contorno menores e, da mesma forma, regiões de interesse (vias) podem apresentar comprimentos de contornos menores que o limiar mínimo e, portanto, são eliminadas. De posse dos comprimentos de contornos de cada região, através de tentativas e erros, é escolhido um limiar cujo valor consiga eliminar o máximo possível de re- giões indesejáveis sem comprometer aquelas correspondentes às vias.

\subsection{Esqueletização}

Supondo que as regiões selecionadas anteriormente correspondem às regiões de vias, a etapa da esqueletização é realizada no intuito de extrair o eixo médio das vias. 0 esqueleto das regiões foi obtido usando o conceito de Transformada do Eixo Médio e adotando a distância euclidiana como descrito na seção 2.3. Na tentativa de eliminar ramificações características do resultado do processo de esqueletização aplicouse a poda, onde as ramificações menores que o parâmetro comprimento são removidas. Essas ramificações podem ser observadas na figura 7B, que mostra o esqueleto da região apresentada na figura 7A. Após a aplicação da poda, usando o valor para o comprimento igual a 20 , tem-se o esqueleto livre das ramificações, como pode ser observado na figura 7C.

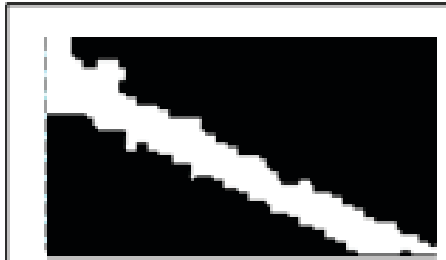

(A)



(B)

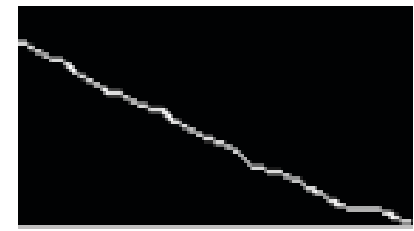

(C)

Figura 7. Esqueletização seguida da poda: (A) região a ser esqueletizada; (B) esqueleto da região e (C) esqueleto da região sem as ramificações.

\subsection{Detecção de Linhas}

0 eixo médio das regiões isoladas anteriormente (seção 3.3) também foi obtido usando o detector de linhas de Steger, sendo necessários alguns parâmetros, tais como o desvio padrão (sigma) que determina a intensidade da suaviza- ção para ser aplicada. Valores grandes deste parâmetro causam uma maior suavização da imagem, podendo levar a uma pior localização da linha. Outros dois parâmetros necessários são os limiares de histerese alto e baixo. O limiar alto é um parâmetro responsável pela permanência das linhas físicas e evita ao máximo as linhas indese- 
jáveis. O limiar baixo complementa as linhas físicas encontradas no plano superior (definido pelo limiar alto) e a continuidade das mesmas é baseada no plano inferior.

Para eliminar algumas linhas espúrias, aplicou-se uma limiarização por comprimento, removendo-se aquelas que possuem um tamanho menor que um limiar definido.

\section{Experimentos e resultados}

Os experimentos foram executados usando recortes dos dados laser de uma região de CuritibaPR, fornecidos pela empresa LACTEC. Trata-se de uma área residencial caracterizada por edificações, ruas pavimentadas, vegetações e áreas de lazer (quadras, praças etc.). Os recortes foram feitos na imagem de intensidade de pulso laser (dados radiométricos). Os resultados dos experimentos foram sobrepostos nas imagens de intensidade e são apresentados e analisados a seguir para cada recorte.

A figura 8 mostra o recorte 1 da imagem de intensidade de pulso laser onde as vias apresentamse mais destacadas que o restante na imagem e com características geométricas regulares. No entanto, verificam-se alguns objetos com níveis de cinza similares aos das vias, como árvores no interior das quadras.

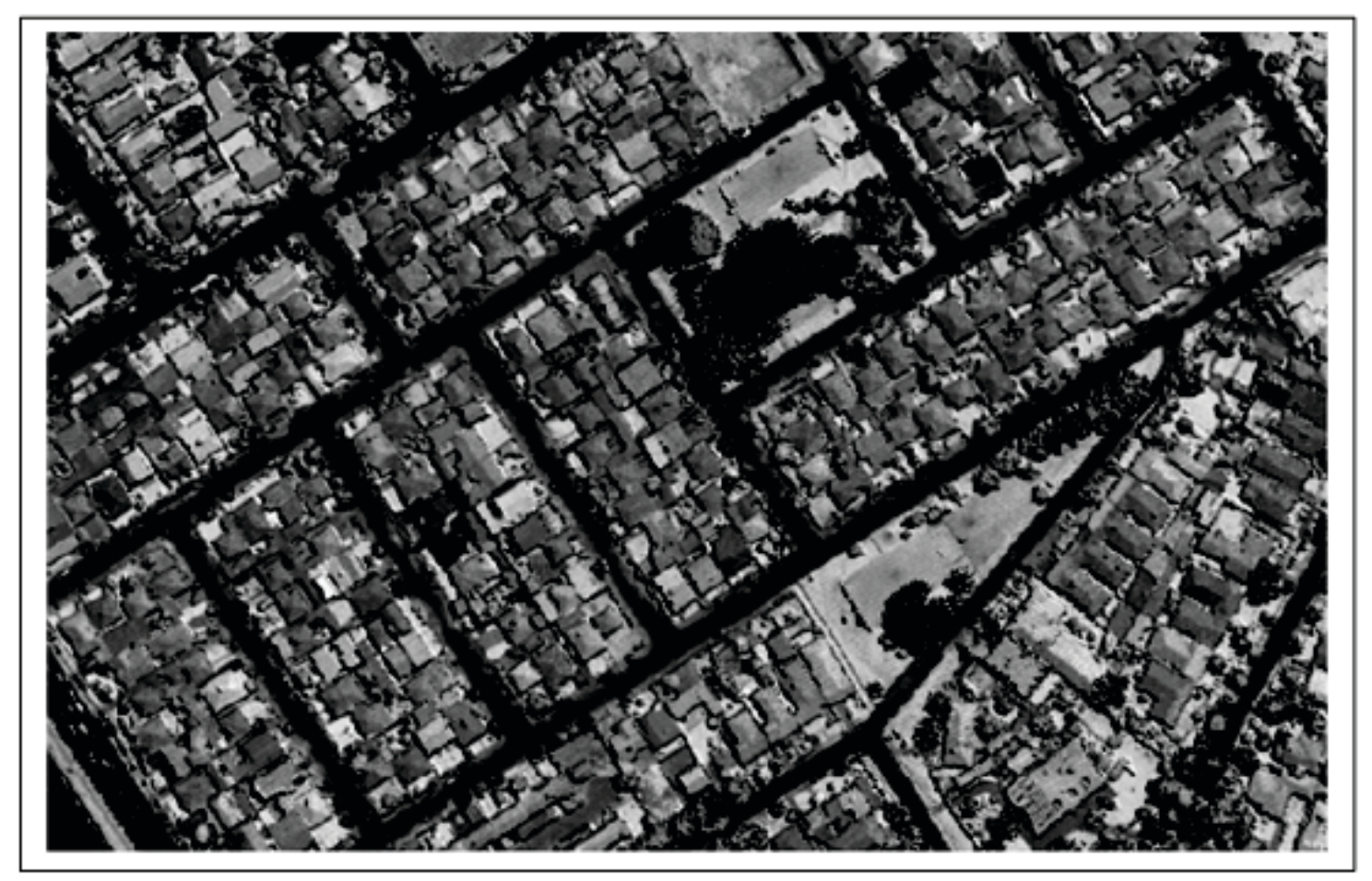

Figura 8. Recorte 1 da imagem de intensidade de pulso laser.

A imagem de intensidade (Fig. 8) foi segmentada utilizando-se um retângulo de dimensão 3x1 (linha x coluna), o valor para a tolerância igual a 4 e o tamanho mínimo de pontos em cada região igual a 100. Os valores usados para estes parâmetros foram obtidos por tentativa e erro, a fim de se chegar a uma segmentação que melhor atendesse o objetivo do trabalho. 0 resultado da segmentação pode ser visualizado na figura 9, onde se observam as vias como regiões alongadas e bordas irregulares. Pode ser observado ainda, que no interior das quadras tem-se uma grande quantidade de regiões menores, correspondentes aos objetos daquele local.

As regiões menores no interior das quadras foram, em sua maioria, eliminadas após a limiariza- ção por comprimento de contorno, como pode ser visto na figura 10 , que mostra as regiões de vias previamente isoladas. No processo de limiarização, duas regiões de vias foram eliminadas (lateral esquerda) e não foi possível eliminar a região correspondente aos objetos escuros no interior das quadras (centro da imagem). Na parte inferior direita da imagem, verifica-se uma via desconectada cuja causa tem origem no processo de segmentação. De fato, a região correspondente apresentavase desconectada de outras regiões, e no processo de limiarização a mesma foi eliminada, pois continha um comprimento de contorno inferior ao limiar escolhido. 0 mesmo problema ocorreu na parte superior esquerda, que apresenta outra via desconectada. 


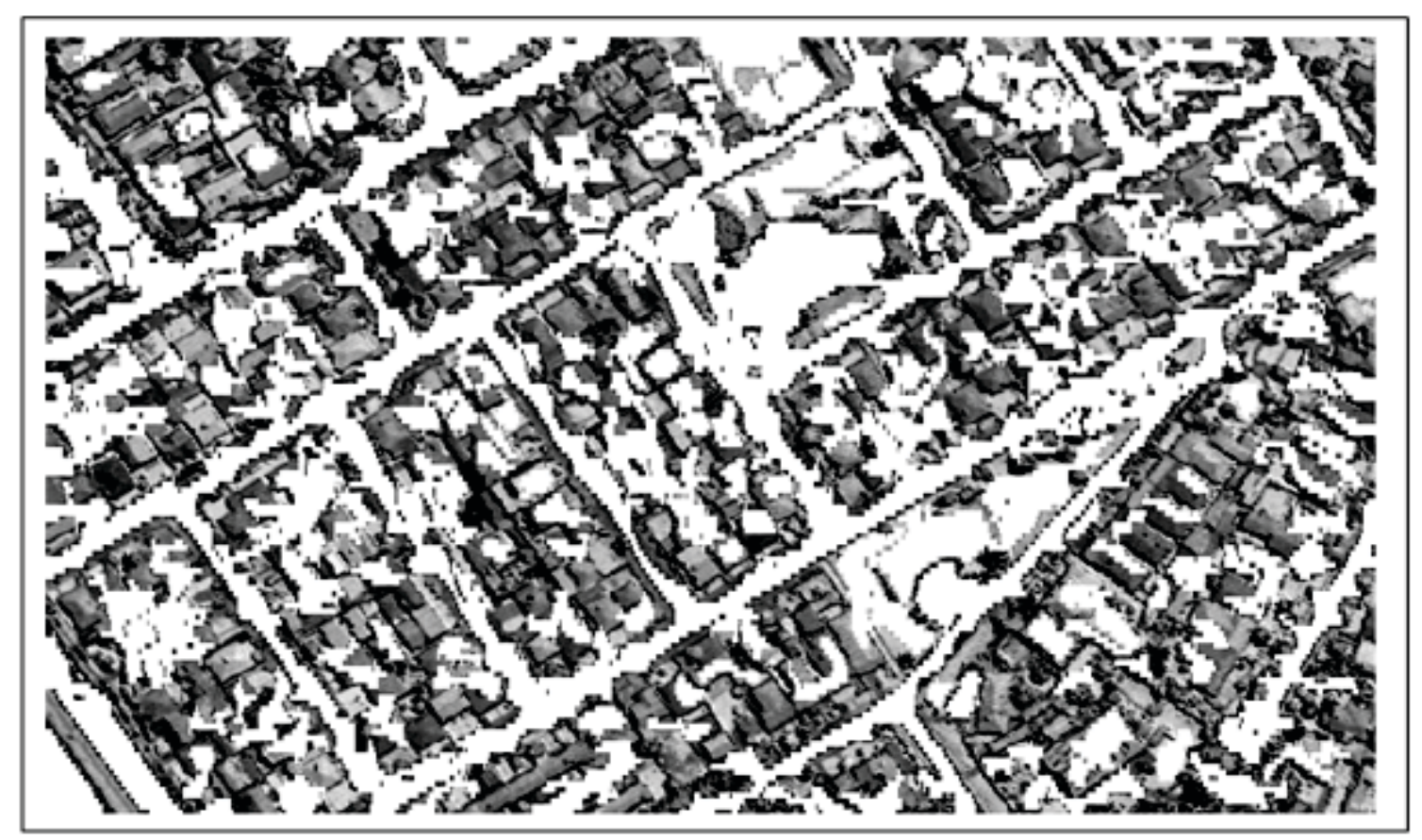

Figura 9. Imagem de intensidade segmentada por crescimento de regiões.

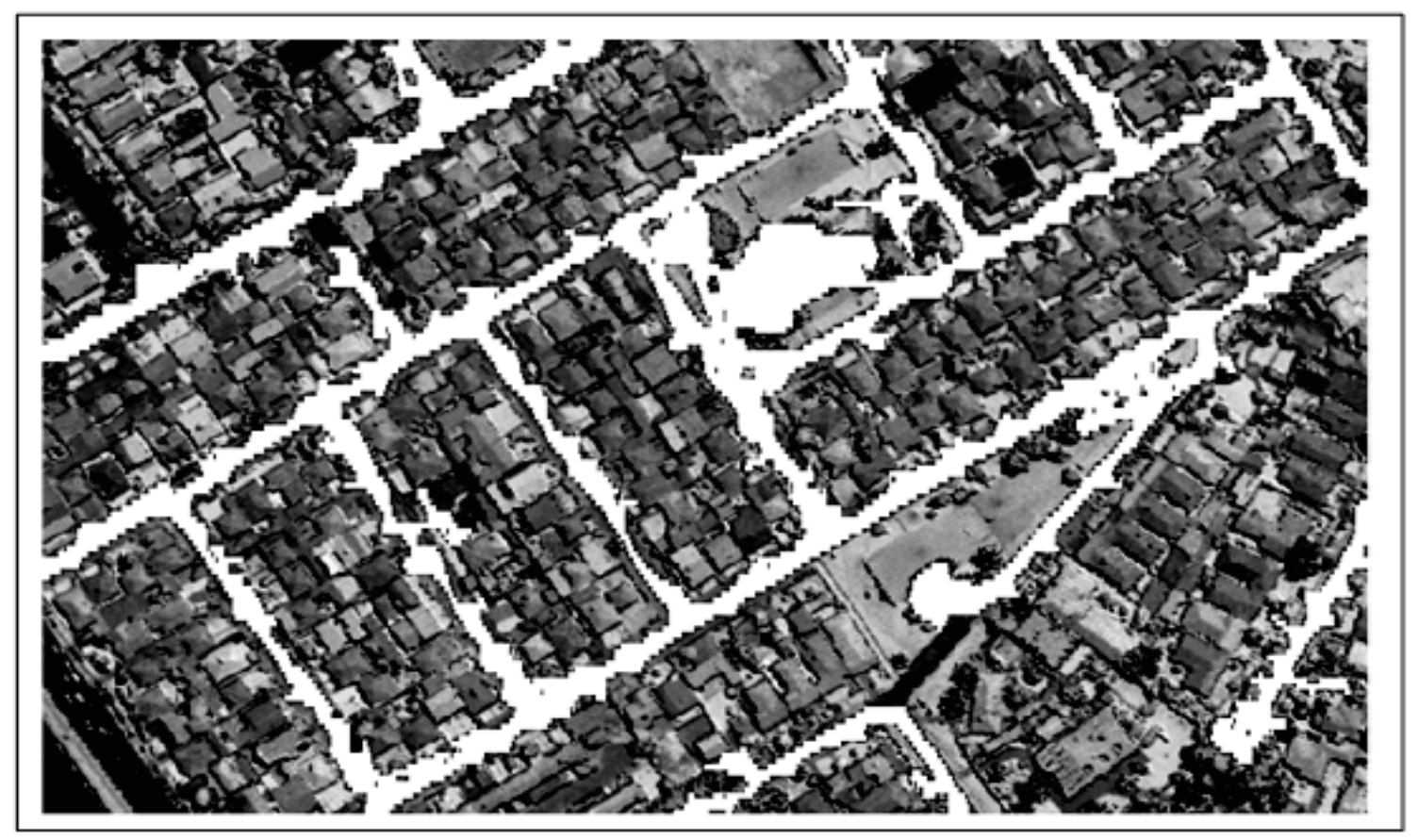

Figura 10. Regiões de vias isoladas.

A figura 11 apresenta o eixo extraído das regiões de vias isoladas através da detecção de linhas usando os seguintes parâmetros: desvio-padrão (sigma) igual a 6 e os limiares de histerese baixo igual a 1 e alto igual a 2. Após a detecção das linhas, aplicouse a limiarização por comprimento. Observando os resultados obtidos, tem-se que os eixos das regiões de vias foram extraídos adequadamente, sendo que somente em alguns casos têm-se algumas linhas indesejadas, causadas por regiões que não puderam ser eliminadas no processo de seleção de regiões de vias (subseção 3.3).

Usando, agora, a esqueletização juntamente com a poda para extrair o eixo das regiões de vias, tem-se o resultado apresentado na figura 12. De uma forma geral, o eixo médio das vias foi obtido com 
sucesso, sendo que somente nas áreas onde as regiões são mais irregulares e não se apresentam muito alongadas é que se verificam algumas ramificações que não puderam ser eliminadas através do método de poda. Alguns pequenos laços formados pelos esqueletos aparecem em diversos lugares. Esses são característicos do processo de esqueletização de regiões que apresentam buracos em seu interior.

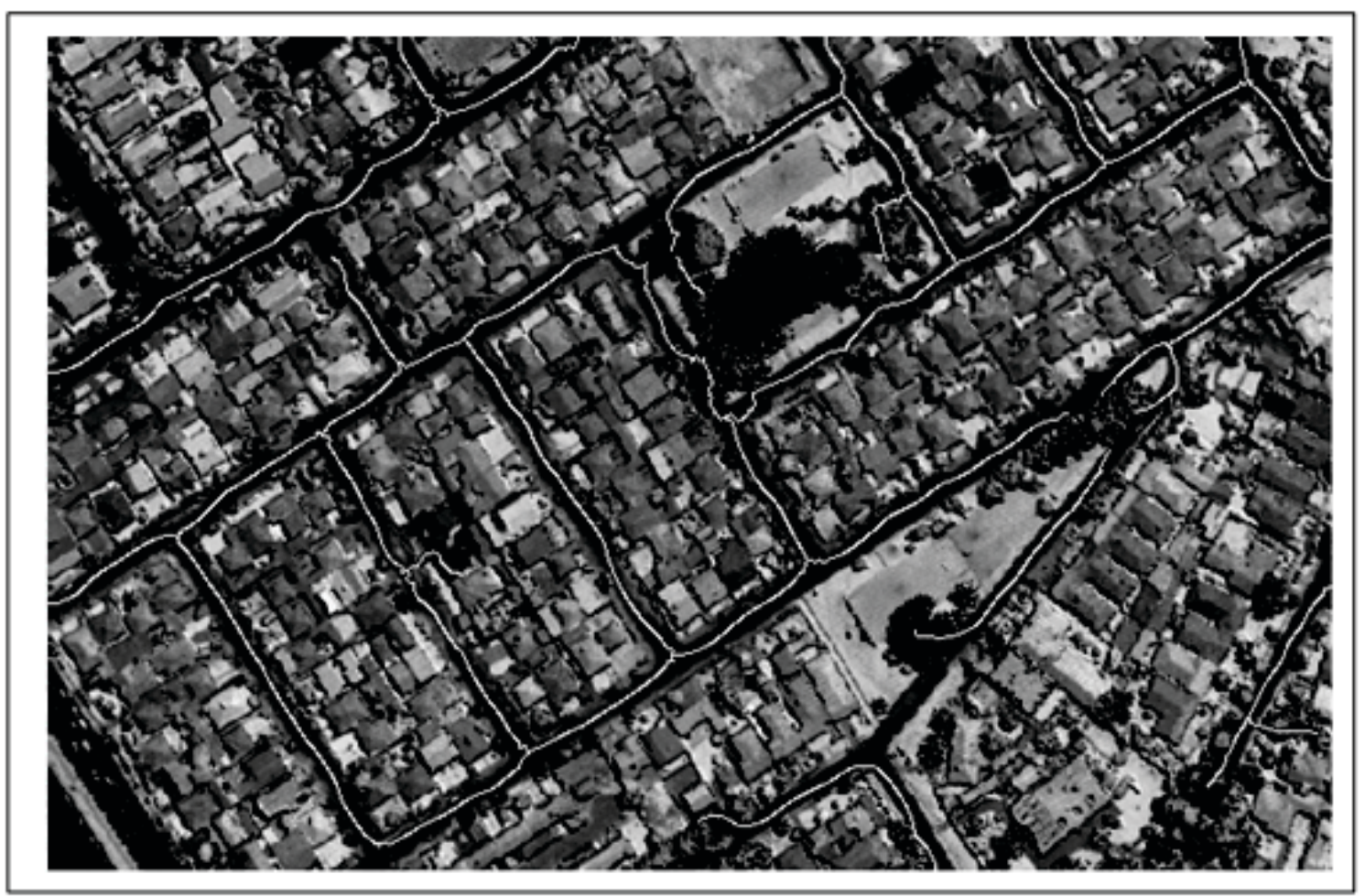

Figura 11. Eixo médio das vias usando detector de linhas de Steger.



Figura 12. Eixo médio das vias usando o método de esqueletização. 
A figura 13 mostra o recorte 2 da imagem de intensidade do pulso laser onde as vias se apresentam com geometria mais complexa, sendo algumas delas obstruídas pela presença de árvores. Na parte superior à direita do recorte é possível verificar uma edificação com estacionamento ao seu redor, que apresenta níveis de cinza muito próximos aos das vias, podendo dificultar a aplicação da metodologia. Nesta mesma região tem-se uma avenida principal e acessos à mesma que se apresentam bem destacados em relação à sua vizinhança. 0 triângulo preto no canto superior direito é o fundo da imagem e não representa nenhum objeto.

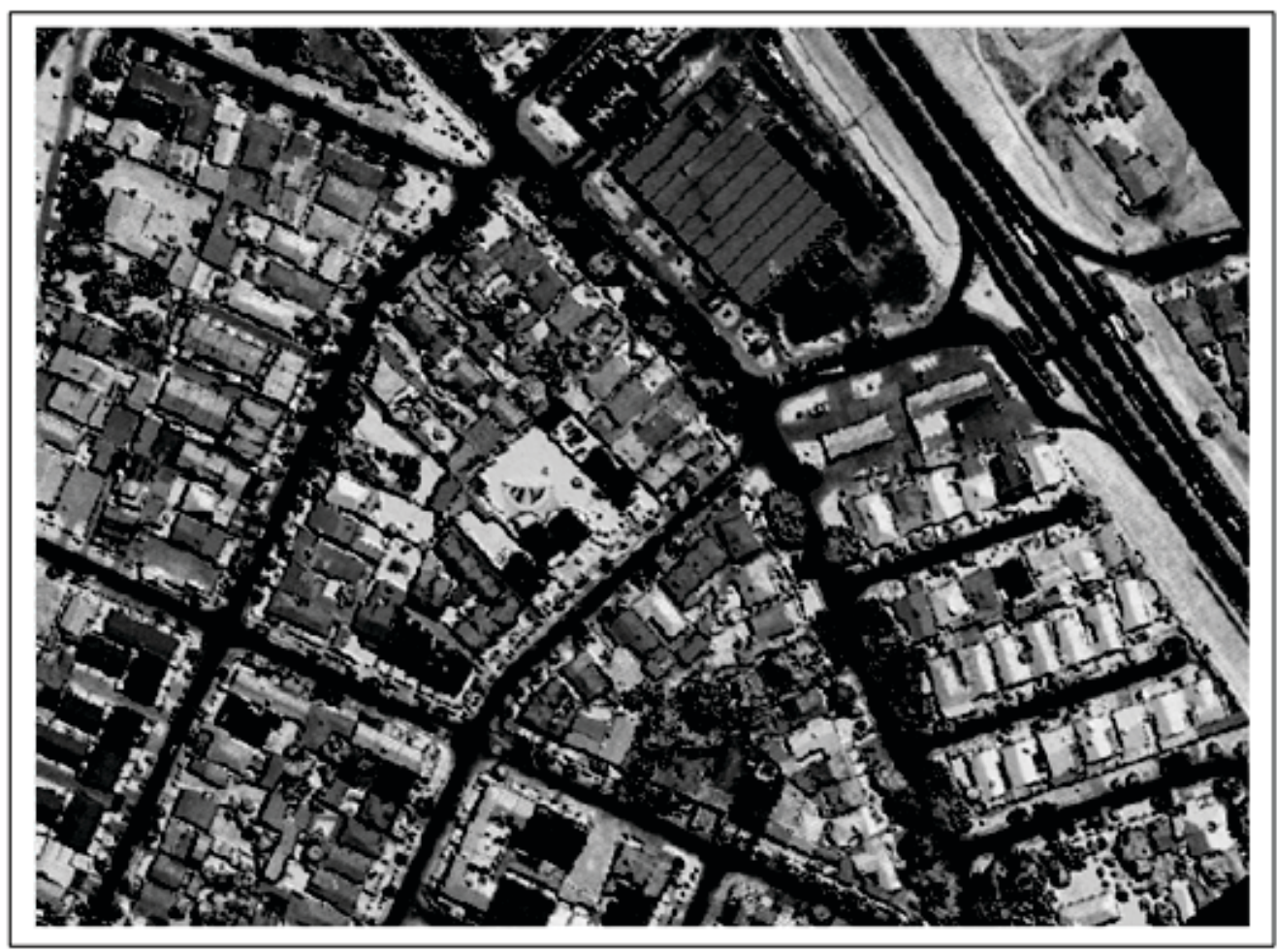

Figura 13. Recorte 2 da imagem de intensidade de pulso laser.

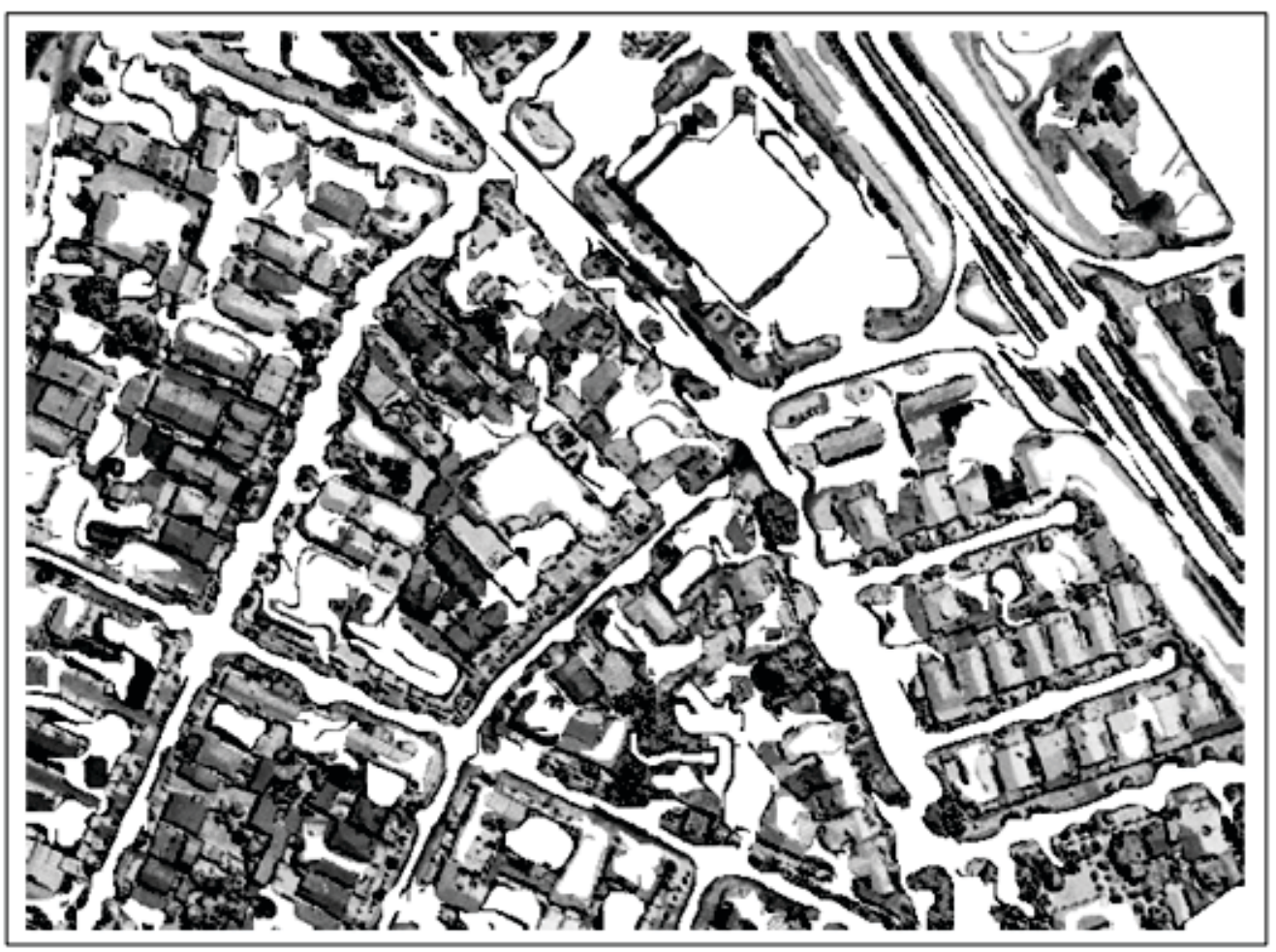

Figura 14. Imagem de intensidade segmentada por crescimento de regiões. 
A imagem de intensidade (Fig. 13) foi segmentada usando um retângulo de dimensão $1 \times 1$ (linha x coluna), a tolerância igual a 2 e o tamanho mínimo de pontos em cada região igual a 200. 0 resultado da segmentação pode ser visualizado na figura 14, que apresenta regiões que representam as vias e também regiões no interior das quadras.

Após a etapa da seleção de regiões de vias, como mostrado na figura 15 , tem-se a maioria das vias isoladas, mas a via de acesso à avenida princi- pal foi eliminada por ser uma região que apresenta um comprimento de contorno bem menor que o limiar dado. Devido a essa limiarização, uma via no inferior da imagem também foi eliminada. Observa-se ainda que o estacionamento na parte superior à direita da imagem foi considerado como via, não sendo possível eliminá-lo através da limiarização por comprimento de contorno das regiões. As vias, de um modo geral, apresentam-se irregulares e com obstruções, dificultando a etapa de detecção do eixo médio da rodovia.



Figura 15. Regiões de vias isoladas.

O eixo das vias foi detectado a partir do método de detecção de linhas de Steger e uma limiarização por comprimento das linhas foi aplicada para eliminar as linhas espúrias, como pode ser visto na figura 16. Para a detecção de linhas foram usados os seguintes parâmetros: desvio-padrão (sigma) igual a 6 e os limiares de histerese baixo igual a 0,5 e alto igual a 1 . As linhas resultantes apresentam-se desconectadas em regiões de vias muito irregulares. Na avenida principal (canto superior direito), apesar da região não apresentar formas muito irregulares, não foi possível detectar o eixo como uma única linha e sim como segmentos de linhas, sendo alguns eliminados pelo processo de limiarização por comprimento.

A esqueletização também foi aplicada na região isolada, detectando os eixos, conforme mostra a figura 17, que apresenta os resultados já após o processo de poda para reduzir as ramificações. Entretanto, muitas delas não foram eliminadas, principalmente aquelas situadas em esqueletos de regiões com formas mais irregulares, como por exemplo, regiões que correspondem ao estacionamento e às vias obstruídas por árvores. $\mathrm{Na}$ verdade, os esqueletos resultantes são bem característicos para regiões com formas irregulares. Os eixos das vias que compõem a avenida principal foram detectados de forma mais contínua, sem muitas falhas quando comparados com o resultado apresentado pela detecção de linhas. No canto superior direito observa-se um esqueleto que foi extraído da região do fundo da imagem, e que não representa qualquer objeto. 




Figura 16. Eixo médio das vias usando o detector de linhas de Steger.

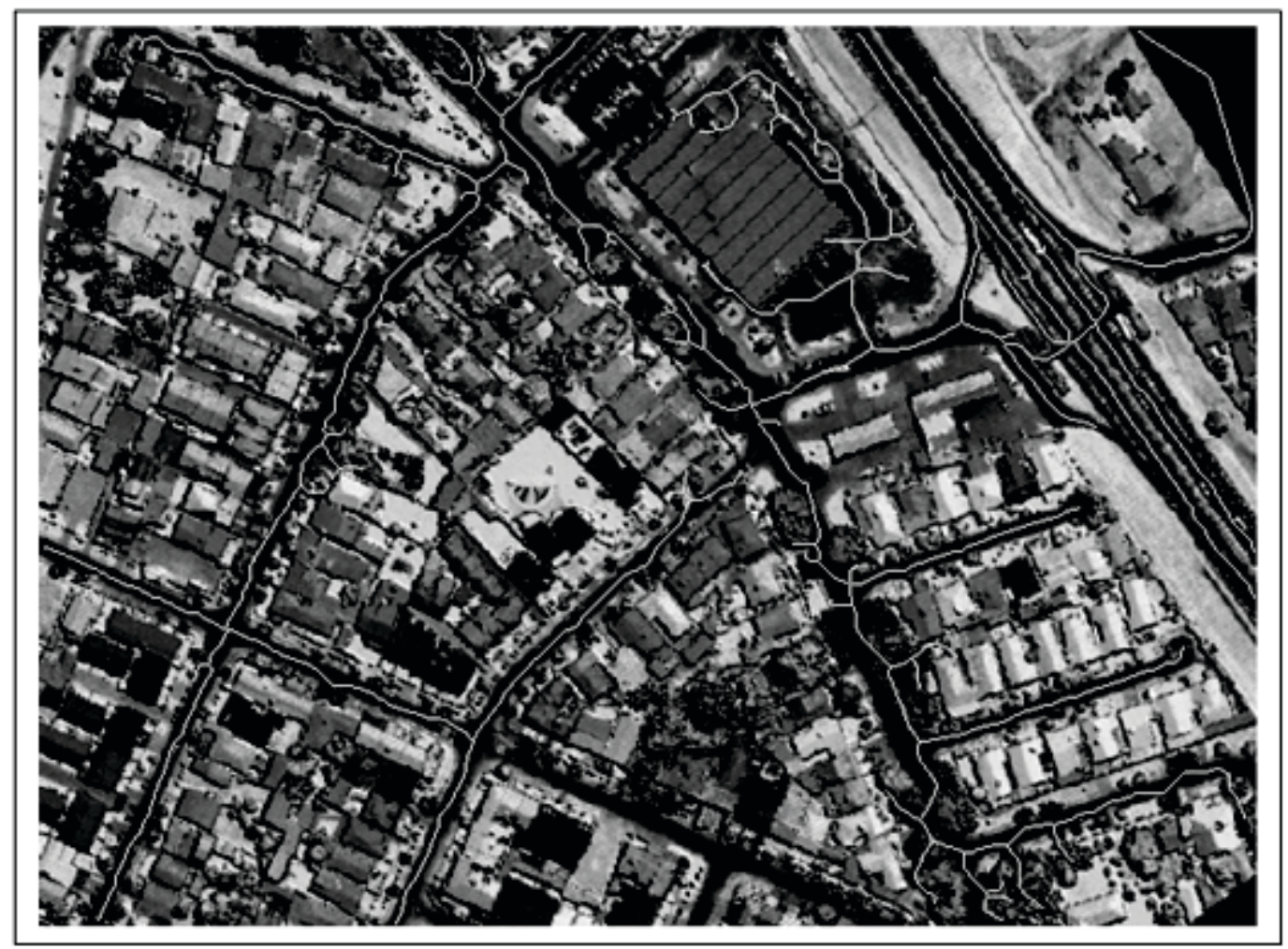

Figura 17. Eixo médio das vias usando o método de esqueletização. 


\section{Conclusões}

0 trabalho proposto é uma primeira investigação de técnicas de análise de imagens que podem ser combinadas e aplicadas nas imagens de intensidade de pulso laser com o intuito de detectar os eixos médios das vias. Primeiramente, as regiões de vias foram detectadas a partir da segmentação da imagem usando o método de crescimento de regiões. A partir dessas regiões detectadas, o eixo médio das vias foi extraído de duas maneiras: como linha usando o conceito de espaço-escala gaussiano, através do detector de linhas de Steger e como esqueleto da região, através da técnica de esqueletização usando o conceito de Transformada do Eixo Médio.

A interpretação visual dos resultados obtidos possibilitou concluir que o eixo médio das vias foi extraído, de uma forma geral, com sucesso. Como era de se esperar, os eixos extraídos são dependentes, principalmente, do resultado apresentado pela segmentação. Neste sentido, métodos de suavização, como o de difusão anisotrópica, que permitam regularizar adequadamente a intensidade das regiões correspondentes aos objetos notáveis da cena, são relevantes para produzir uma segmentação com um mínimo de fragmentação. Em relação à limiarização por comprimento de contorno das regiões, verificou-se que é impossível eliminar todas as regiões indesejadas, pois estas podem apresentar um comprimento de contorno maior que algumas regiões de vias. 0 mesmo ocorreu com a limiarização por comprimento de linhas extraídas pelo método de Steger. De uma forma geral, para a detecção do eixo das vias, o método de detecção de linhas de Steger apresentou melhor resultado quando comparado com o método de esqueletização. Na verdade, irregularidades nas bordas das regiões influenciam em demasia o esqueleto resultante.

Os resultados obtidos mostraram que os métodos propostos cumpriram com adequação o objetivo de detectar vias usando somente as imagens de intensidade do pulso laser, de forma a explorar o máximo da característica de alto contraste que as vias possuem nessa imagem, embora com algumas desvantagens quanto ao caráter empírico, onde os valores dos parâmetros são obtidos por tentativa e erro.

A combinação da imagem de intensidade com dados de altura do laser pode certamente contribuir para melhorar a qualidade dos resultados, bem como reduzir a dependência de um operador no fornecimento de vários parâmetros dos métodos propostos. Por este motivo, esta é uma das direções para pesquisa futura. Outros métodos de suavização também deverão ser estudados e comparados com a filtragem anisotrópica. Métodos morfológicos poderão também ser empregados para reduzir as irregularidades presentes nas regiões resultantes do processo de segmentação.

Agradecimentos - Este artigo é resultado de apoio do $\mathrm{CNPq}$ através de bolsa de doutorado (Edital MCT/CNPq no 27/2007) e de bolsa de Produtividade em Pesquisa (PQ). Os dados laser usados neste artigo foram fornecidos pela LACTEC.

\section{Referências}

Alharthy, A. \& Bethel, J. 2003. Automated Road Extraction from LIDAR Data. In: PROCEEDINGS OF ASPRS ANNUAL CONFERENCE, 2003, Anchorage. Proceedings... Anchorage.

Barcelos, C.A.Z., Boaventura, M. \& Silva Jr., E.C. 2003. A Well-balanced Flow Equation for Noise Removal and Edge Detection. IEEE Transactions on Image Processing, 12 (7): 751-764.

Blum, H.1967. A transformation for extracting new descriptors of shape. In: WATHEN-DUNN, W. MODELS FOR PERCEPTIONS OF SPEECH AND VISUAL FORMS, 1967, Cambridge, MIT Press, p. 362-380.

Clode, S., Kootsookos, P. \& Rottensteiner, F. 2004. The automatic extraction of roads from LIDAR data. In: THE INTERNATIONAL ARCHIVES OF THE PHOTOGRAMMETRY, REMOTE SENSING AND SPATIAL INFORMATION SCIENCE, 2004, Istanbul, Comm. III/3, p. 231-237.

Clode, S., Rottensteiner, F., Kootsookos, P. \& Zelniker, E. 2007. Detection and vectorisation of roads from LIDAR data. Photogrammetric Engineering and Remote Sensing, 73(5): 517-535.

Dal Poz, A.P. 2001. Semi-automatic road extraction methods by dynamic programming optimization and a cooperative road floower. The Photogrammetric Journal of Finland, 17(2): 13-22.

Gonzalez, R.C. \& Woods, R.E. 2008. Digital Image Processing. $3^{\text {th }}$ Edition. Prentice Hall, $954 \mathrm{p}$.

Hinz, S. \& Baumgartner, A. 2003.Automatic extraction of urban road networks from multi-view aerial imagery. ISPRS Journal of Photogrammetry and Remote Sensing, 58(1-2): 83-98.

Hu, X., Tao, C.V. \& Hu, Y. 2004. Automatic road extraction from dense urban area by integrated processing of high resolution imagery and LIDAR data. In: THE INTERNATIONAL ARCHIVES OF PHOTOGRAMMETRY, REMOTE SENSING AND SPATIAL INFORMATION SCIENCES, 2004, Istanbul, Comm. IIII/3, p.320-325. 
Ishibashi, R. 2008. Extração de segmentos de rodovias em imagens de resoluções variadas usando o princípio de bordas paralelas. Presidente Prudente. 136p. Dissertação de Mestrado, Programa de Pósgraduação em Ciências Cartográficas, Universidade Estadual Paulista.

Malik, J. \& Perona, P.1990. Scale space and edge detection using anisotropic diffusion. IEEE Transactions on Pattern Analysis and Machine Intelligence, 12 (7): 629639.

Plotze, R.O. \& Bruno, O.M. 2004. Estudo e comparação de algoritmos de esqueletonização para imagens binárias. In: IV CONGRESSO BRASILEIRO DE COMPUTAÇÃO CBCOMP2004, 2004, Itajaí. Anais... Itajaí, p. 59-64.

Samadzadegan, F., Hahn, M. \& Bigdeli, B. 2009.Automatic Road Extraction from LIDAR Data based on Classifier Fusion. Urban Remote Sensing Joint Event.

Soille, P. 1999. Morphological Image Analysis. Berlin, Springer-Verlag, $316 \mathrm{p}$.

Steger, C. 1998. An Unbiased Detector of Curvilinear Structures. IEEE Transactions on Pattern Analysis and Machine Intelligence, 20(2): 113-125.

Steger, C. 2000. Subpixel - Precise Extraction of Lines and Edges. International Society for Photogrammetry and Remote Sensing, 33: 141-156.

Tiwari, P.S., Pande, H. \& Pandey, A.K. 2009. Automatic urban road extraction using Airborne Laser Scanning/altimetry and high resolution satellite data. Journal of the Indian Society of Remote Sensing, 37(2): 223-231.

Vale, G.M., Galvanin, E.A.S. \& Dal Poz, A.P.O. 2004. Detector de Canny-EDP: Uma Combinação entre as Teorias de Canny e de Difusão Anisotrópica Não Linear. Revista Brasileira de Cartografia, 56(02): 156168.

Wehr, A. \& Lohr, U. 1999. Airborne laserscanning - an introduction and overview. In: ISPRS Journal of Photogrammetry and Remote Sensing, 1999. Proceedings... v. 54(2-3), p.68-82.

Zhang, C. 2004.Towards an operational system for automated updating of road databases by integration of imagery and geodata. ISPRS International Journal of Photogrammetry and Remote Sensing, 58: 166-186.

Zhu, P., Lu, Z., Chen, X., Honda, K. \& Eiumnoh, A. 2004.Extraction of city roads through shadow path reconstruction using LASER data. Photogrammetric Engineering and Remote Sensing, 70(12): 1433-1440. 
